PROCEEDINGS OF THE

AMERICAN MATHEMATICAL SOCIETY

Volume 135, Number 3, March 2007, Pages 695-704

S 0002-9939(06)08537-6

Article electronically published on September 15, 2006

\title{
GROWTH OF RELATIVELY HYPERBOLIC GROUPS
}

\author{
XIANGDONG XIE \\ (Communicated by Alexander N. Dranishnikov)
}

\begin{abstract}
We show that a finitely generated group that is hyperbolic relative to a collection of proper subgroups either is virtually cyclic or has uniform exponential growth.
\end{abstract}

\section{INTRODUCTION}

Let $G$ be a finitely generated group, and $S$ a finite generating set. Denote by $d_{S}$ the word metric on $G$ with respect to $S$ and by $\beta(G, S, k)$ the number of elements of $G$ a $d_{S}$ distance at most $k$ from the identity. The exponential growth rate of $G$ with respect to $S$ is $\omega(G, S)=\lim _{k \rightarrow \infty}(\beta(G, S, k))^{1 / k}$. Notice that the limit exists due to the submultiplicativity: $\beta(G, S, m+n) \leq \beta(G, S, m) \beta(G, S, n)$. We say $G$ has exponential growth if $\omega(G, S)>1$ for some (hence any) finite generating set $S$; and we say $G$ has uniform exponential growth if $\omega(G)=\inf _{S} \omega(G, S)>1$, where $S$ varies over all finite generating sets of $G$.

J. Wilson constructed groups that have exponential growth but not uniform exponential growth $([\mathrm{W}])$. On the other hand, among the following classes of groups, exponential growth implies uniform exponential growth: linear groups over fields with zero characteristic ([EMO $)$, hyperbolic groups $([\underline{\mathrm{K}}]$ ), one-relator groups (GD]), solvable groups ([02]), and geometrically finite groups acting on pinched Hadamard manifolds $([\mathrm{AN}])$. In this paper we show that the same is true for relatively hyperbolic groups.

Theorem 1.1. Let $G$ be a finitely generated group that is hyperbolic relative to a collection of proper subgroups. Then $G$ either has uniform exponential growth or has a finite index cyclic subgroup.

Relatively hyperbolic groups are generalizations of Gromov hyperbolic groups. Typical examples of relatively hyperbolic groups include Gromov hyperbolic groups, fundamental groups of finite volume real hyperbolic manifolds, and groups acting properly and cocompactly on spaces with isolated flats $([\mathrm{HK}])$. There are five different definitions for relatively hyperbolic groups: one due to each of Gromov $([\mathrm{G}])$, B. Farb $([\mathrm{F}])$ and D. Osin ([O1] $)$, and two due to B. Bowditch $([\mathrm{B}])$. These definitions are all equivalent for finitely generated groups. However, we remark that Osin's definition is also valid for infinitely generated groups. We shall use

Received by the editors April 10, 2005 and, in revised form, October 18, 2005.

2000 Mathematics Subject Classification. Primary 20F65.

Key words and phrases. Exponential growth, uniform exponential growth, relatively hyperbolic groups, geometrically finite groups.

(C)2006 American Mathematical Society Reverts to public domain 28 years from publication 
Bowditch's definition of relatively hyperbolic groups as geometrically finite groups. The main ingredient in our proof is Bowditch's theorem on the existence of an invariant collection of disjoint horoballs (see Proposition 2.2).

The usual way for proving uniform exponential growth is as follows: for any finite generating set $S$ of $G$, find two elements $g_{1}, g_{2} \in G$ with word length bounded independent of $S$, such that $\left\langle g_{1}, g_{2}\right\rangle$ is free with basis $\left\{g_{1}, g_{2}\right\}$. We shall use the same strategy. Let $G$ be a relatively hyperbolic group and $S$ a finite generating set. For any positive integer $n$, let $S(n)=\left\{g \in G: d_{S}(g, i d) \leq n\right\}$. Notice that $S(n)$ is also a finite generating set of $G$, and $S(n) \subset S(m)$ if $n \leq m$. In particular, $S \subset S(n)$ for all $n \geq 1$.

We prove uniform exponential growth in two steps:

Step 1: There exists some positive integer $n_{0}$ with the following property. For any finite generating set $S$ of $G, S\left(n_{0}\right)$ contains a hyperbolic element (see Proposition 5.1).

Step 2: There exists some positive integer $k_{0}$ with the following property. If a finite generating set $S$ of $G$ contains a hyperbolic element, then there are $g_{1}, g_{2} \in$ $S\left(k_{0}\right)$ such that $\left\langle g_{1}, g_{2}\right\rangle$ is free with basis $\left\{g_{1}, g_{2}\right\}$ (see Corollary 3.2 and Proposition 4.1).

It should be noted that uniform exponential growth for geometrically finite groups acting on pinched Hadamard manifolds has been established by R. Alperin and G. Noskov ( $\underline{\mathrm{AN}})$. Some of our arguments are similar to theirs. Theorem 1.1 was conjectured by C. Drutu in D.

It was pointed out by the referee that our proof actually establishes a stronger result: any non-elementary relatively hyperbolic group is uniformly non-amenable in the sense of Osin (

Notation. For a metric space $Y$, any subset $A \subset Y$, and any $r \geq 0$, we denote by $N_{r}(A)=\{y \in Y: d(y, A) \leq r\}$ the closed $r$-neighborhood of $A$. For any $p, q \in Y$, $p q$ denotes a geodesic segment between $p$ and $q$, although it is not unique in general.

\section{Relatively HYPERBOLIC GROUPS AS GEOMETRICALLY FINITE GROUPS}

Here we recall the notion of geometrically finite groups and Bowditch's result on the existence of an invariant system of disjoint horoballs (see Proposition 2.2). The reader is referred to $\underline{B}$ for more details.

Suppose that $M$ is a compact metrizable topological space, and a group $G$ acts by homeomorphisms on $M$. We say that $G$ is a convergence group if the induced action on the space of distinct triples is properly discontinuous. Let $G$ be a convergence group on $M$. A point $\xi \in M$ is a conical limit point if there exists a sequence in $G$, $\left\{g_{n}\right\}_{n=1}^{\infty}$, and two points $\zeta \neq \eta \in M$, such that $g_{n}(\xi) \rightarrow \zeta$ and $g_{n}\left(\xi^{\prime}\right) \rightarrow \eta$ for all $\xi^{\prime} \neq \xi$. An element $g \in G$ is a hyperbolic element if it has infinite order and fixes exactly two points in $M$. A subgroup $H<G$ is parabolic if it is infinite, fixes a point $\xi \in M$, and contains no hyperbolic elements. In this case, the fixed point of $H$ is unique and is referred to as a parabolic point. The nontrivial elements in a parabolic subgroup are called parabolic elements. A parabolic point $\xi$ is a bounded parabolic point if its stabilizer $\operatorname{Stab}(\xi):=\{g \in G: g(\xi)=\xi\}$ acts properly and cocompactly on $M \backslash\{\xi\}$. A convergence group $G$ on $M$ is a geometrically finite group if each point of $M$ is either a conical limit point or a bounded parabolic point. 
Definition 2.1. A group $G$ is hyperbolic relative to a family of finitely generated subgroups $\mathcal{G}$ if it acts properly discontinuously by isometries, on a proper geodesic hyperbolic space $X$, such that the induced action on $\partial X$ is of convergence, geometrically finite, and such that the maximal parabolic subgroups are exactly the elements of $\mathcal{G}$.

By definition a relatively hyperbolic group is a geometrically finite group. A. Yaman $([\mathrm{Y}]$ ) proved that if $G$ is a geometrically finite group on a perfect metrizable compact space $M$, and the maximal parabolic subgroups are finitely generated, then $G$ is hyperbolic relative to the family of maximal parabolic subgroups.

Now let $X$ be a $\delta$-hyperbolic geodesic metric space for some $\delta \geq 0$. Recall that each geodesic triangle in $X$ is $\delta$-thin, that is, each edge is contained in the $\delta$-neighborhood of the union of the other two edges. Below we shall denote by $c=c(\delta)$ a constant that depends only on $\delta$. Let $\xi \in \partial X$. A (not necessarily continuous) function $h: X \rightarrow \mathbb{R}$ is a horofunction about $\xi$ if there are constants $c_{1}=c_{1}(\delta), c_{2}=c_{2}(\delta)$ such that if $x, a \in X$ and $d(a, x \xi) \leq c_{1}$ for some geodesic ray $x \xi$ from $x$ to $\xi$, then $|h(a)-(h(x)+d(x, a))| \leq c_{2}$. A closed subset $B \subset X$ is a horoball about $\xi$ if there is a horofunction $h$ about $\xi$ and a constant $c=c(\delta)$ such that $h(x) \geq-c$ for all $x \in B$, and $h(x) \leq c$ for all $x \in X \backslash B$. Note that $\xi$ is uniquely determined by $B$, and we refer to it as the center of the horoball.

Proposition 2.2 (B. Bowditch, Proposition 6.13 in [B]). Let $G$ be a relatively hyperbolic group, and $X$ a proper hyperbolic geodesic metric space that $G$ acts upon as in Definition 2.1. Let $\Pi$ be the set of all bounded parabolic points in $\partial X$. Then $\Pi / G$ is finite. Moreover, for any $r \geq 0$, there is a collection of horoballs $\mathcal{B}=\left\{B_{\xi}\right.$ : $\xi \in \Pi\}$ indexed by $\Pi$ with the following properties:

(1) $\mathcal{B}$ is r-separated, that is, $d\left(B_{\xi}, B_{\eta}\right) \geq r$ for all $\xi \neq \eta \in \Pi$;

(2) $\mathcal{B}$ is $G$-invariant, that is, $g\left(B_{\xi}\right)=B_{g(\xi)}$ for all $g \in G$ and $\xi \in \Pi$;

(3) $Y(\mathcal{B}) / G$ is compact, where $Y(\mathcal{B})=X \backslash \bigcup_{\xi \in \Pi}$ int $\left(B_{\xi}\right)$.

\section{Axes of hyperbolic Elements}

In this section we study how a hyperbolic element "translates" its "axes".

Let $G$ and $X$ be as in Definition 2.1. That is, $X$ is a proper $\delta$-hyperbolic geodesic space for some $\delta \geq 0$ and $G$ acts properly discontinuously by isometries on $X$, such that the induced action on $\partial X$ is of convergence and geometrically finite.

Recall that, in a $\delta$-hyperbolic space $X$, any two complete geodesics that have the same endpoints in $\partial X$ have Hausdorff distance at most $2 \delta$. For a hyperbolic element $\gamma \in G$, let $\gamma_{+}$and $\gamma_{-}$be the attracting and repelling fixed points of $\gamma$ in $\partial X$ respectively. We shall call any complete geodesic with $\gamma_{+}, \gamma_{-}$as endpoints an axis of $\gamma$, and denote by $A_{\gamma}$ the union of all axes of $\gamma$. Note that $\gamma$ may have many different axes and an axis of $\gamma$ in general is not invariant under $\gamma$. However, for any axis $c$ of $\gamma, \gamma(c)$ is also an axis of $\gamma$ and hence the Hausdorff distance between $c$ and $\gamma(c)$ is at most $2 \delta$.

Proposition 2.2 implies that there is a $200 \delta$-separated $G$-invariant collection of horoballs $\mathcal{B}$ centered at the parabolic points such that $Y(\mathcal{B}) / G$ is compact.

Lemma 3.1. There exists a positive integer $k_{1}$ with the following property: for any infinite order element $\gamma \in G$ and any $x \in Y(\mathcal{B})$, there is some $k, 1 \leq k \leq k_{1}$, such that $d\left(\gamma^{k}(x), x\right) \geq 200 \delta$. 
Proof. Notice that the action of $G$ on $Y(\mathcal{B})$ is properly discontinuous and cocompact. It follows that there is some integer $k_{1} \geq 1$, such that for any $x \in Y(\mathcal{B})$, the cardinality of $\{g \in G: d(x, g(x)) \leq 200 \delta\}$ is less than $k_{1}$. In particular, for any $x \in Y(\mathcal{B})$ and any infinite order element $\gamma \in G$, there is some $k, 1 \leq k \leq k_{1}$, such that $d\left(\gamma^{k}(x), x\right) \geq 200 \delta$.

Corollary 3.2. If a finite generating set $S$ of $G$ contains a hyperbolic element, then there is a hyperbolic element $\gamma \in S\left(k_{1}\right)$ and some $x \in A_{\gamma}$ such that $d(\gamma(x), x) \geq$ $200 \delta$, where $k_{1}$ is the constant in Lemma 3.1 .

Proof. Let $g \in S$ be a hyperbolic element and $c$ an axis of $g$. It follows from the definition of a horoball that $c$ is not contained in any horoball. Since $\mathcal{B}$ is a disjoint collection of horoballs and $c$ is connected, we have $c \cap Y(\mathcal{B}) \neq \emptyset$. Let $x \in c \cap Y(\mathcal{B})$. By Lemma 3.1, there is some $k, 1 \leq k \leq k_{1}$, such that $d\left(g^{k}(x), x\right) \geq 200 \delta$. Notice that $g^{k} \in S\left(k_{1}\right)$ is hyperbolic and $c$ is also an axis of $g^{k}$.

For a complete geodesic $c$ in $X$, we define a map $P_{c}: X \rightarrow c$ as follows: for any $x \in X$, let $P_{c}(x) \in c$ be a point with $d\left(x, P_{c}(x)\right)=d(x, c)$. Note that for any two points $x_{1}, x_{2} \in c$ with $d\left(x, x_{1}\right)=d\left(x, x_{2}\right)=d(x, c)$, we have $d\left(x_{1}, x_{2}\right) \leq 4 \delta$.

Lemma 3.3. Let $c, c^{\prime}: \mathbb{R} \rightarrow X$ be two geodesics with $c(+\infty)=c^{\prime}(+\infty)$ and $c(-\infty)=c^{\prime}(-\infty)$. Let $a, b \in \mathbb{R}$, with $b \geq a+8 \delta$, and $a^{\prime}, b^{\prime} \in \mathbb{R}$ be determined by $c^{\prime}\left(a^{\prime}\right)=P_{c^{\prime}}(c(a))$ and $c^{\prime}\left(b^{\prime}\right)=P_{c^{\prime}}(c(b))$. Then $\left(b^{\prime}-a^{\prime}\right) \geq(b-a)-4 \delta$.

Proof. Recall that the Hausdorff distance between $c$ and $c^{\prime}$ is at most $2 \delta$. It follows from the triangle inequality that $\left|b^{\prime}-a^{\prime}\right| \geq(b-a)-4 \delta$. So either $\left(b^{\prime}-a^{\prime}\right) \geq(b-a)-4 \delta$ or $\left(b^{\prime}-a^{\prime}\right) \leq-(b-a)+4 \delta$. Suppose $\left(b^{\prime}-a^{\prime}\right) \leq-(b-a)+4 \delta$. Let $x_{i}=c(b+i \delta)$, $i=0,1, \cdots$, and $y_{i}=P_{c^{\prime}}\left(x_{i}\right)$. Then $y_{0}=c^{\prime}\left(b^{\prime}\right), y_{1}, \cdots$ is a sequence of points on $c^{\prime}$ with $d\left(y_{i}, y_{i+1}\right) \leq 5 \delta$. Since $\left(b^{\prime}-a^{\prime}\right) \leq-(b-a)+4 \delta \leq-4 \delta$ and $y_{i} \rightarrow c(+\infty)$, there is some $i \geq 1$ such that $d\left(y_{i}, c^{\prime}\left(a^{\prime}\right)\right) \leq 2.5 \delta$. The triangle inequality implies that $(b+i \delta)-a=d\left(x_{i}, c(a)\right) \leq d\left(x_{i}, y_{i}\right)+d\left(y_{i}, c^{\prime}\left(a^{\prime}\right)\right)+d\left(c^{\prime}\left(a^{\prime}\right), c(a)\right) \leq 6.5 \delta$, contradicting the fact that $b \geq a+8 \delta$.

Lemma 3.4. Let $g \in G$ be a hyperbolic element, and $c$ an axis of $g$. Suppose $x \in c$ is a point with $d(g(x), x) \geq 20 \delta$. Then for any integers $i<j$, the point $P_{c}\left(g^{j}(x)\right)$ lies between $P_{c}\left(g^{i}(x)\right)$ and $g_{+}$.

Proof. Denote $x_{i}=P_{c}\left(g^{i}(x)\right)$. Note that $x_{i} \rightarrow g_{+}$as $i \rightarrow+\infty$. It suffices to show that for any $i, x_{i}$ lies between $x_{i-1}$ and $x_{i+1}$. Set $t=d(g(x), x)$. The triangle inequality implies that $t-4 \delta \leq d\left(x_{i}, x_{i+1}\right) \leq t+4 \delta$. Suppose $x_{i}$ does not lie between $x_{i-1}$ and $x_{i+1}$ for some $i$. Then $d\left(x_{i-1}, x_{i+1}\right) \leq 8 \delta$ and $d\left(g^{i-1}(x), g^{i+1}(x)\right) \leq 12 \delta$. Since $g$ is an isometry, $d\left(x, g^{2}(x)\right) \leq 12 \delta$. We shall show that $d\left(x, g^{2}(x)\right) \geq 2 t-16 \delta$, which is a contradiction.

Let $\sigma: \mathbb{R} \rightarrow X$ be the parameterization of $c$ with $\sigma(0)=x$ and $\sigma(+\infty)=g_{+}$, $\sigma(-\infty)=g_{-}$. Then $g \circ \sigma$ is the parameterization of $g(c)$ with $g \circ \sigma(0)=g(x)$ and $g \circ \sigma(+\infty)=g_{+}, g \circ \sigma(-\infty)=g_{-}$. Since $t-4 \delta \leq d\left(x_{1}, x\right) \leq t+4 \delta$, we have $x_{1}=\sigma(b)$ with either $-(t+4 \delta) \leq b \leq-(t-4 \delta)$ or $t-4 \delta \leq b \leq t+4 \delta$. We consider the case $-(t+4 \delta) \leq b \leq-(t-4 \delta)$, the other case can be handled similarly. Let $y_{1}=P_{c}\left(g\left(x_{1}\right)\right)$. Then $y_{1}=\sigma(a)$ for some $a \in \mathbb{R}$. Now Lemma 3.3 applied to $\sigma, g \circ \sigma$ and the projections of $g(x), g\left(x_{1}\right)$ onto $c$ implies that $b-a \geq 0-b-4 \delta \geq t-8 \delta$. It follows that $d\left(y_{1}, x\right)=0-a \geq-b+t-8 \delta \geq 2 t-12 \delta$. By the triangle inequality $d\left(x, g^{2}(x)\right) \geq d\left(x, y_{1}\right)-d\left(y_{1}, g\left(x_{1}\right)\right)-d\left(g\left(x_{1}\right), g^{2}(x)\right) \geq 2 t-12 \delta-2 \delta-2 \delta=2 t-$ $16 \delta$. 
Lemma 3.5. Let $g \in G$ be a hyperbolic element. Suppose there is a point $x \in A_{g}$ with $d(x, g(x)) \geq 200 \delta$. Then $|d(y, g(y))-d(z, g(z))| \leq 40 \delta$ for all $y, z \in A_{g}$.

Proof. Let $c$ be an axis of $g$ that contains $x$. We first show $|d(y, g(y))-d(x, g(x))| \leq$ $16 \delta$ for all $y \in c$. Fix $y \in c$. Denote $t=d(x, g(x)), x_{i}=P_{c}\left(g^{i}(x)\right), x_{i}^{\prime}=P_{c}\left(g\left(x_{i}\right)\right)$ and $y^{\prime}=P_{c}(g(y))$. Then $d\left(x_{i}, g^{i}(x)\right) \leq 2 \delta$ and $d\left(x_{i}, x_{i-1}\right) \geq t-4 \delta$. By Lemma 3.4 there is some $i$ such that $y$ lies between $x_{i}$ and $x_{i+1}$, and $x_{i+1}$ lies between $x_{i}$ and $g_{+}$. The triangle inequality implies

$$
\left|d\left(y^{\prime}, x_{i-1}^{\prime}\right)-d\left(y, x_{i-1}\right)\right|=\left|d\left(y^{\prime}, x_{i-1}^{\prime}\right)-d\left(g(y), g\left(x_{i-1}\right)\right)\right| \leq 4 \delta
$$

and

$$
d\left(x_{i}, x_{i-1}^{\prime}\right) \leq d\left(x_{i}, g^{i}(x)\right)+d\left(g^{i}(x), g\left(x_{i-1}\right)\right)+d\left(g\left(x_{i-1}\right), x_{i-1}^{\prime}\right) \leq 6 \delta .
$$

It follows that $\left|d\left(y^{\prime}, x_{i}\right)-d\left(y, x_{i-1}\right)\right| \leq 10 \delta$. In particular, $d\left(y^{\prime}, x_{i}\right) \geq d\left(y, x_{i-1}\right)-$ $10 \delta=d\left(y, x_{i}\right)+d\left(x_{i}, x_{i-1}\right)-10 \delta \geq d\left(y, x_{i}\right)+t-4 \delta-10 \delta>d\left(y, x_{i}\right)$. On the other hand, Lemma 3.3 applied to $g(c), c$ and the projections of $g(y), g\left(x_{i-1}\right)$ onto $c$ implies that $y^{\prime}$ lies between $x_{i}$ and $g_{+}$. It now follows from $d\left(y^{\prime}, x_{i}\right)>d\left(y, x_{i}\right)$ that $y$ lies between $x_{i}$ and $y^{\prime}$. Now

$$
\begin{aligned}
\left|d\left(y, y^{\prime}\right)-d\left(x_{i-1}, x_{i}\right)\right| & =\left|\left[d\left(x_{i}, y\right)+d\left(y, y^{\prime}\right)\right]-\left[d\left(x_{i-1}, x_{i}\right)+d\left(x_{i}, y\right)\right]\right| \\
& =\left|d\left(x_{i}, y^{\prime}\right)-d\left(x_{i-1}, y\right)\right| \leq 10 \delta .
\end{aligned}
$$

Finally

$$
\begin{aligned}
|d(y, g(y))-d(x, g(x))|= & \left|d(y, g(y))-d\left(g^{i-1}(x), g^{i}(x)\right)\right| \\
\leq & \left|d\left(y, y^{\prime}\right)-d\left(x_{i-1}, x_{i}\right)\right|+d\left(g(y), y^{\prime}\right) \\
& +d\left(g^{i-1}(x), x_{i-1}\right)+d\left(g^{i}(x), x_{i}\right) \\
\leq & 16 \delta .
\end{aligned}
$$

Now fix any $y \in A_{g}$. Set $y^{\prime}=P_{c}(y) \in c$. Then $|d(y, g(y))-d(x, g(x))| \leq$ $\left|d\left(y^{\prime}, g\left(y^{\prime}\right)\right)-d(x, g(x))\right|+d\left(y, y^{\prime}\right)+d\left(g\left(y^{\prime}\right), g(y)\right) \leq 20 \delta$. By the triangle inequality, we have $|d(y, g(y))-d(z, g(z))| \leq 40 \delta$ for all $y, z \in A_{g}$.

\section{Free subgroups}

The goal in this section is to find two short hyperbolic elements that generate a free group.

Proposition 4.1. Let $G$ be a relatively hyperbolic group, and let $X$ be a proper $\delta$-hyperbolic geodesic metric space that $G$ acts upon as in Definition 2.1. Then there exists a positive integer $k_{2}$ with the following property. If $S$ is a finite generating set of $G$ and $s \in S$ is a hyperbolic element such that $d(s(x), x) \geq 200 \delta$ for some $x \in A_{s}$, then there are $g_{1}, g_{2} \in S\left(k_{2}\right)$ such that $\left\langle g_{1}, g_{2}\right\rangle$ is free with basis $\left\{g_{1}, g_{2}\right\}$.

4.1. Ping-Pong lemma. The proof of Proposition 4.1 is based on the following Ping-Pong lemma.

Lemma 4.2. Let $G$ be a group acting on a set $X$, and $g_{1}, g_{2}$ two elements of $G$. If $X_{1}, X_{2}$ are disjoint subsets of $X$ and for all $n \neq 0, i \neq j, g_{i}^{n}\left(X_{j}\right) \subset X_{i}$, then the subgroup $\left\langle g_{1}, g_{2}\right\rangle$ is free with basis $\left\{g_{1}, g_{2}\right\}$.

We will apply the Ping-Pong lemma in the following setting. 
Lemma 4.3. Let $X$ be a metric space and $g_{1}, g_{2}$ isometries of $X$. Let $B_{i} \subset A_{i} \subset X$ $(i=1,2)$ be subsets of $X$, and $p_{i}: X \rightarrow A_{i}$ a map. Denote $X_{i}=p_{i}^{-1}\left(A_{i}-B_{i}\right)$. Then the assumptions in the Ping-Pong lemma are satisfied if the following conditions hold:

(1) $X_{1} \cap X_{2}=\emptyset$;

(2) $g_{i}^{n}\left(p_{i}^{-1}\left(B_{i}\right)\right) \subset X_{i}$ for all $n \neq 0, i=1,2$.

In our case, $g_{i}$ is a hyperbolic element, $A_{i}$ is an axis of $g_{i}, B_{i} \subset A_{i}$ is a segment, and $p_{i}=P_{A_{i}}$.

4.2. Two hyperbolic elements. In this section we prove Proposition 4.1 For this, we shall find two short hyperbolic elements and segments in their axes such that the two conditions in Lemma 4.3 are satisfied.

Let $\mathcal{B}$ be a fixed $200 \delta$-separated $G$-invariant collection of horoballs centered at the parabolic points. Recall (see the proof of Lemma 3.1) that there is an integer $k_{1} \geq 1$ such that for any $x \in Y(\mathcal{B})$, the cardinality of $\{g \in G: d(x, g(x)) \leq 200 \delta\}$ is less than $k_{1}$.

Lemma 4.4. Let $g \in G$ be a hyperbolic element such that there is some $x \in A_{g}$ with $d(x, g(x)) \geq 200 \delta$, and $\gamma \in G$ an element such that $\left\{\gamma\left(g_{+}\right), \gamma\left(g_{-}\right)\right\} \cap\left\{g_{+}, g_{-}\right\}=\emptyset$. Let $c_{1}$ and $c_{2}$ be axes of $g$ and $h:=\gamma g \gamma^{-1}$ respectively. Let $a=\min \{t \in \mathbb{R}$ : $\left.d\left(c_{1}(t), c_{2}\right) \leq 2 \delta\right\}$ and $b=\max \left\{t \in \mathbb{R}: d\left(c_{1}(t), c_{2}\right) \leq 2 \delta\right\}$, and denote $y=c_{1}(a)$, $z=c_{1}(b)$. Then $d(y, z) \leq 3 k_{1} d(x, g(x))$.

Proof. Denote $t=d(x, g(x))$. Suppose $d(y, z)>3 k_{1} t$. Let $y^{\prime}=c_{2}\left(a^{\prime}\right) \in c_{2}$, $z^{\prime}=c_{2}\left(b^{\prime}\right) \in c_{2}$ with $d\left(y, y^{\prime}\right), d\left(z, z^{\prime}\right) \leq 2 \delta$. It is easy to see from the $\delta$-thin condition that the Hausdorff distance between $y z$ and $y^{\prime} z^{\prime}$ is at most $4 \delta$. After replacing $h$ by its inverse if necessary, we may assume $g$ and $h$ translate in the "same direction", that is, if both $c_{1}$ and $c_{2}$ are parameterized from the repelling fixed point toward the attracting fixed point, then $b^{\prime}>a^{\prime}$ (we have $b>a$ by definition).

Since $h$ is a conjugate of $g$ or its inverse, Lemma 3.5 implies that the inequality $\left|d\left(p, g^{i}(p)\right)-d\left(q, h^{i}(q)\right)\right| \leq 40 \delta$ holds for all $i \geq 1$, and all $p \in A_{g}, q \in A_{h}$. In particular, $\left|d\left(y, g^{i}(y)\right)-d\left(y^{\prime}, h^{i}\left(y^{\prime}\right)\right)\right| \leq 40 \delta$ for all $i \geq 1$.

For $i \geq 0$, let $y_{i}=P_{c_{1}}\left(g^{i}(y)\right)$ and $y_{i}^{\prime}=P_{c_{2}}\left(h^{i}\left(y^{\prime}\right)\right)$. Lemma 3.4 implies that $y_{i+1}$ lies between $y_{i}$ and $c_{1}(+\infty)$. Since $d\left(y_{i}, y_{i+1}\right) \leq d(y, g(y))+4 \delta \leq d(x, g(x))+40 \delta+$ $4 \delta \leq 2 t$, we have $y_{i} \in y z$ for all $1 \leq i \leq k_{1}$. Similarly $y_{i}^{\prime} \in y^{\prime} z^{\prime}$ for all $1 \leq i \leq k_{1}$. Hence for $1 \leq i \leq k_{1}$, there exists $z_{i} \in y z$ with $d\left(z_{i}, y_{i}^{\prime}\right) \leq 4 \delta$.

We first assume $y \in Y(\mathcal{B})$. The triangle inequality implies $\left|d\left(y, y_{i}\right)-d\left(y^{\prime}, y_{i}^{\prime}\right)\right| \leq$ $\left|d\left(y, g^{i}(y)\right)-d\left(y^{\prime}, h^{i}\left(y^{\prime}\right)\right)\right|+d\left(y_{i}^{\prime}, h^{i}\left(y^{\prime}\right)\right)+d\left(y_{i}, g^{i}(y)\right) \leq 40 \delta+2 \delta+2 \delta=44 \delta$ and $\left|d\left(y^{\prime}, y_{i}^{\prime}\right)-d\left(y, z_{i}\right)\right| \leq d\left(y, y^{\prime}\right)+d\left(z_{i}, y_{i}^{\prime}\right) \leq 6 \delta$. It follows that

$$
d\left(y_{i}, z_{i}\right)=\left|d\left(y, y_{i}\right)-d\left(y, z_{i}\right)\right| \leq 50 \delta
$$

and

$$
\begin{aligned}
d\left(g^{i}(y), h^{i}\left(y^{\prime}\right)\right) & \leq d\left(g^{i}(y), y_{i}\right)+d\left(y_{i}, z_{i}\right)+d\left(z_{i}, y_{i}^{\prime}\right)+d\left(y_{i}^{\prime}, h^{i}\left(y^{\prime}\right)\right) \\
& \leq 2 \delta+50 \delta+4 \delta+2 \delta=58 \delta .
\end{aligned}
$$

Now for any $i, 1 \leq i \leq k_{1}$, we have

$$
\begin{aligned}
d\left(y, h^{-i} \circ g^{i}(y)\right) & =d\left(h^{i}(y), g^{i}(y)\right) \leq d\left(h^{i}(y), h^{i}\left(y^{\prime}\right)\right)+d\left(h^{i}\left(y^{\prime}\right), g^{i}(y)\right) \\
& =d\left(y, y^{\prime}\right)+d\left(h^{i}\left(y^{\prime}\right), g^{i}(y)\right) \leq 60 \delta .
\end{aligned}
$$


Since $y \in Y(\mathcal{B})$, it follows from the definition of $k_{1}$ that there are $i \neq j, 1 \leq i, j \leq k_{1}$, with $h^{-i} \circ g^{i}=h^{-j} \circ g^{j}$. Consequently $h^{j-i}=g^{j-i}$, contradicting the fact that $g$ and $h$ do not share any fixed points in $\partial X$.

Now suppose $y \notin Y(\mathcal{B})$. Since $Y(\mathcal{B})$ is $G$-invariant, $g(y) \notin Y(\mathcal{B})$. There are horoballs $B_{1}, B_{2} \in \mathcal{B}$ with $y \in B_{1}$ and $g(y) \in B_{2}$. Note $B_{1} \neq B_{2}$, otherwise $g\left(B_{1}\right)=B_{1}$ and so $g$ fixes the center of $B_{1}$, contradicting the fact that $g$ is a hyperbolic element. Since $\mathcal{B}$ is $200 \delta$-separated and $d\left(y_{1}, g(y)\right) \leq 2 \delta$, there is some $p \in c_{1}$ between $y$ and $y_{1}$ such that $p \in Y(\mathcal{B})$. Now we run the argument from the pervious paragraph using $p$ instead of $y$.

Remark 4.1. The proof of Lemma 4.4 actually shows that for any $\gamma \in G$, either $\left\{\gamma\left(g_{+}\right), \gamma\left(g_{-}\right)\right\}=\left\{g_{+}, g_{-}\right\}$or $\left\{\gamma\left(g_{+}\right), \gamma\left(g_{-}\right)\right\} \cap\left\{g_{+}, g_{-}\right\}=\emptyset$ holds.

Now let $S$ be a finite generating set of $G$ and suppose $s \in S$ is a hyperbolic element such that $d(s(x), x) \geq 200 \delta$ for some $x \in A_{s}$. If $\left\{s^{\prime}\left(s_{+}\right), s^{\prime}\left(s_{-}\right)\right\}=\left\{s_{+}, s_{-}\right\}$ for all $s^{\prime} \in S$, then $A_{s}$ is invariant under $G$. In this case, $G$ is 2-ended and hence is virtually infinite cyclic.

We now assume there is some $\gamma \in S$ with $\left\{\gamma\left(s_{+}\right), \gamma\left(s_{-}\right)\right\} \cap\left\{s_{+}, s_{-}\right\}=\emptyset$. Let $c$ be an axis of $s$. Set $g_{1}=s^{10 k_{1}}$ and $g_{2}=\gamma g_{1} \gamma^{-1}, A_{1}=c, A_{2}=\gamma(c), p_{1}=P_{c}$ and $p_{2}=P_{\gamma(c)}$. If $d\left(A_{1}, A_{2}\right)>2 \delta$, let $p \in A_{1}, q \in A_{2}$ with $d(p, q)=d\left(A_{1}, A_{2}\right)$; in this case, let $B_{1} \subset A_{1}$ be the closed segment with midpoint $p$ and length $40 \delta$ and similarly for $B_{2} \subset A_{2}$. If $d\left(A_{1}, A_{2}\right) \leq 2 \delta$, let $a=\min \left\{t \in \mathbb{R}: d\left(c(t), A_{2}\right) \leq 2 \delta\right\}$ and $b=\max \left\{t \in \mathbb{R}: d\left(c(t), A_{2}\right) \leq 2 \delta\right\}$, and denote $y=c(a), z=c(b)$. Also let $y^{\prime}, z^{\prime} \in A_{2}$ with $d\left(y, y^{\prime}\right) \leq 2 \delta$ and $d\left(z, z^{\prime}\right) \leq 2 \delta$; in this case, let $B_{1} \subset A_{1}$ be the closed $20 \delta$-neighborhood of $y z$ in $A_{1}$ and let $B_{2} \subset A_{2}$ be the closed $20 \delta$ neighborhood of $y^{\prime} z^{\prime}$ in $A_{2}$. We shall show that the two conditions in Lemma 4.3 are satisfied, and hence Proposition 4.1 holds.

Lemma 4.5. Condition (2) of Lemma 4.3 is satisfied.

Proof. We only write down the proof in the case $d\left(A_{1}, A_{2}\right) \leq 2 \delta, i=1$ and $n>0$; the other cases are similar or simpler.

Let $y \in p_{1}^{-1}\left(B_{1}\right)$. We need to show $p_{1}\left(g_{1}^{n}(y)\right) \notin B_{1}$. Denote $z=p_{1}(y), y^{\prime}=$ $g_{1}^{n}(y), z^{\prime}=g_{1}^{n}(z)$ and $y^{\prime \prime}=p_{1}\left(y^{\prime}\right)$. Lemma 3.5 implies $d(z, s(z)) \geq 160 \delta$. Set $t=d(z, s(z))$. By Lemma 3.4 and the fact that $d\left(p_{1}\left(s^{i}(z)\right), p_{1}\left(s^{i+1}(z)\right)\right) \geq t-4 \delta$, we obtain $d\left(p_{1}\left(z^{\prime}\right), z\right) \geq 10 k_{1} n(t-4 \delta)$.

Let $z^{\prime \prime}=p_{1}\left(z^{\prime}\right)$. We claim $d\left(y^{\prime \prime}, z^{\prime \prime}\right) \leq 13 \delta$. We first finish the proof assuming the claim. The claim implies $d\left(z, y^{\prime \prime}\right) \geq 10 k_{1}(t-4 \delta) n-13 \delta$. Note Lemma3.5implies $t-$ $4 \delta \geq 2 d(x, s(x)) / 5$. It follows that $d\left(z, y^{\prime \prime}\right) \geq 4 n k_{1} d(x, s(x))-13 \delta \geq 4 k_{1} d(x, s(x))-$ $13 \delta>3 k_{1} d(x, s(x))+40 \delta \geq \operatorname{length}\left(B_{1}\right)$. The last inequality follows from the definition of $B_{1}$ and Lemma 4.4 Since $z=p_{1}(y) \in B_{1}$, we have $p_{1}\left(g_{1}^{n}(y)\right)=y^{\prime \prime} \notin$ $B_{1}$.

We now prove the claim. We may assume $d\left(y^{\prime \prime}, z^{\prime \prime}\right)>3 \delta$. Fix geodesic segments $z^{\prime} z^{\prime \prime}, y^{\prime} z^{\prime \prime}$ and $y^{\prime} y^{\prime \prime}$. Let $p \in y^{\prime \prime} z^{\prime \prime}$ with $d\left(p, y^{\prime \prime}\right)=3 \delta$. We use the $\delta$-thin condition. Consider the geodesic triangle $\left(y^{\prime} y^{\prime \prime} z^{\prime \prime}\right)$. There is some $q \in y^{\prime} y^{\prime \prime} \cup y^{\prime} z^{\prime \prime}$ with $d(p, q) \leq$ $\delta$. If $q \in y^{\prime} y^{\prime \prime}$, then $d\left(q, y^{\prime \prime}\right) \geq d\left(p, y^{\prime \prime}\right)-d(p, q) \geq 2 \delta$ and hence $d(q, p)<d\left(q, y^{\prime \prime}\right)$, contradicting the fact that $y^{\prime \prime}=p_{1}\left(y^{\prime}\right)$. So $q \in y^{\prime} z^{\prime \prime}$. Now consider the geodesic triangle $\left(y^{\prime} z^{\prime \prime} z^{\prime}\right)$. There is some $r \in z^{\prime} z^{\prime \prime} \cup z^{\prime} y^{\prime}$ with $d(q, r) \leq \delta$. If $r \in z^{\prime} z^{\prime \prime}$, then $d\left(r, z^{\prime \prime}\right) \leq d\left(z^{\prime}, z^{\prime \prime}\right) \leq 2 \delta$ and hence $d\left(y^{\prime \prime}, z^{\prime \prime}\right)=d\left(y^{\prime \prime}, p\right)+d\left(p, z^{\prime \prime}\right) \leq 3 \delta+$ $d(p, q)+d(q, r)+d\left(r, z^{\prime \prime}\right) \leq 7 \delta$. Suppose $r \in y^{\prime} z^{\prime}$. Then $d\left(r, z^{\prime}\right)=d\left(r, g_{1}^{n}\left(A_{1}\right)\right) \leq$ 
$d(r, p)+d\left(p, g_{1}^{n}\left(A_{1}\right)\right) \leq d(r, q)+d(q, p)+2 \delta \leq 4 \delta$. Therefore $d\left(z^{\prime \prime}, p\right) \leq d\left(z^{\prime \prime}, z^{\prime}\right)+$ $d\left(z^{\prime}, r\right)+d(r, p) \leq 8 \delta$ and $d\left(z^{\prime \prime}, y^{\prime \prime}\right) \leq 11 \delta$.

The following lemma can be easily seen using approximating trees (GhD $)$. For completeness, here we include a proof using only the $\delta$-thin condition.

Lemma 4.6. Condition (1) of Lemma 4.3 is satisfied.

Proof. We give the proof for the case $d\left(A_{1}, A_{2}\right)>2 \delta$; the proof when $d\left(A_{1}, A_{2}\right) \leq 2 \delta$ is similar.

We prove by contradiction. Assume $d\left(A_{1}, A_{2}\right)>2 \delta$ and $z \in X_{1} \cap X_{2}$, where $X_{i}=p_{i}^{-1}\left(A_{i}-B_{i}\right)$. Without loss of generality, we may assume $d\left(z, A_{2}\right) \leq d\left(z, A_{1}\right)$. We claim that there is some $q^{\prime} \in A_{2}$ with $d\left(p_{1}(z), q^{\prime}\right) \leq 8.2 \delta$.

We first finish the proof of the lemma assuming the above claim. Fix geodesic segments $q^{\prime} p_{1}(z), p q^{\prime}$ and $p q$. Let $m \in A_{1}$ be the midpoint of $p_{1}(z) p \subset A_{1}$. Since $p_{1}(z) \notin B_{1}$, we have $d\left(p_{1}(z), p\right)>20 \delta$ and $d(m, p)=d\left(m, p_{1}(z)\right)>10 \delta$. The $\delta$-thin condition applied to the triangle $\left(p, q^{\prime}, p_{1}(z)\right)$ shows that there is some $n \in$ $p q^{\prime} \cup q^{\prime} p_{1}(z)$ with $d(m, n) \leq \delta$. Since $d\left(p_{1}(z), q^{\prime}\right) \leq 8.2 \delta$ and $d\left(m, p_{1}(z)\right)>10 \delta$, the point $n$ must lie on $p q^{\prime}$. Now the $\delta$-thin condition applied to the triangle $\left(p, q, q^{\prime}\right)$ shows that there is some $x \in p q \cup q q^{\prime}$ with $d(n, x) \leq \delta$. If $x \in q q^{\prime} \subset A_{2}$, then $d(m, x) \leq d(m, n)+d(n, x) \leq 2 \delta$, contradicting the assumption that $d\left(A_{1}, A_{2}\right)>2 \delta$. Hence $x \in p q$. Since $p \in A_{1}$ and $q \in A_{2}$ realize the distance between $A_{1}$ and $A_{2}$, we have $d(x, p)=d\left(x, A_{1}\right)$; it follows that $d(x, p) \leq d(x, n)+d(n, m) \leq 2 \delta$. Consequently, $d(p, m) \leq d(p, x)+d(x, n)+d(n, m) \leq 4 \delta$, contradicting $d(m, p)>$ $10 \delta$.

Now we prove the claim. Recall that $d\left(z, A_{2}\right) \leq d\left(z, A_{1}\right)$. Since $d\left(A_{1}, A_{2}\right)>2 \delta$, we have $d\left(z, A_{1}\right)>\delta$. Pick $y \in z p_{1}(z)$ with $\delta<d\left(y, p_{1}(z)\right) \leq 1.1 \delta$. Fix geodesic segments $z p_{1}(z), z p, z q$ and $z p_{2}(z)$. The $\delta$-thin condition applied to the triangle $\left(z, p, p_{1}(z)\right)$ shows that there is some $w_{1} \in p_{1}(z) p \cup p z$ with $d\left(y, w_{1}\right) \leq \delta$. Since $d\left(z, p_{1}(z)\right)=d\left(z, A_{1}\right)$ and $y \in z p_{1}(z)$ with $d\left(y, p_{1}(z)\right)>\delta$, we must have $w_{1} \in p z$. Similarly there is some $w_{2} \in q z \cup p q$ with $d\left(w_{1}, w_{2}\right) \leq \delta$. The argument in the preceding paragraph shows $w_{2} \in q z$. The $\delta$-thin condition then shows that there is some $w_{3} \in q p_{2}(z) \cup p_{2}(z) z$ with $d\left(w_{2}, w_{3}\right) \leq \delta$. If $w_{3} \in q p_{2}(z)$, then $d\left(w_{3}, p_{1}(z)\right) \leq$ $d\left(w_{3}, w_{2}\right)+d\left(w_{2}, w_{1}\right)+d\left(w_{1}, y\right)+d\left(y, p_{1}(z)\right) \leq 4.1 \delta$, and the claim holds. Assume $w_{3} \in z p_{2}(z)$. Then $d\left(w_{3}, z\right) \geq d(z, y)-d\left(y, w_{1}\right)-d\left(w_{1}, w_{2}\right)-d\left(w_{2}, w_{3}\right) \geq d(z, y)-$ $3 \delta=d\left(z, p_{1}(z)\right)-d\left(y, p_{1}(z)\right)-3 \delta \geq d\left(z, p_{1}(z)\right)-4.1 \delta \geq d\left(z, p_{2}(z)\right)-4.1 \delta$. It follows that $d\left(w_{3}, p_{2}(z)\right) \leq 4.1 \delta$. Consequently, $d\left(p_{2}(z), p_{1}(z)\right) \leq d\left(p_{2}(z), w_{3}\right)+$ $d\left(w_{3}, p_{1}(z)\right) \leq 8.2 \delta$. The proof of the claim is complete.

\section{EXISTENCE OF HYPERBOLIC ELEMENTS}

In this section we establish the following result that guarantees the existence of hyperbolic elements with short word length.

Proposition 5.1. Let $G$ be an infinite relatively hyperbolic group and $X$ a $\delta$ hyperbolic geodesic metric space that $G$ acts upon as in Definition 2.1. Then there exists a positive integer $n_{0}$ with the following property: for any finite generating set $S$ of $G, S\left(n_{0}\right)$ contains a hyperbolic element.

For a finite set of isometries $F$ of a metric space $X$ and $x \in X$, we let $\lambda(x, F)=$ $\max \{d(f(x), x): f \in F\}$. We use the following result of M. Koubi $([\mathbf{K}])$. 
Proposition 5.2. Let $X$ be a $\delta$-hyperbolic geodesic metric space, and $G$ a group of isometries of $X$ with finite generating set $S$. If $\lambda(x, S)>100 \delta$ for all $x \in X$, then $G$ contains a hyperbolic element $g$ such that $d_{S}(i d, g)=1$ or 2 .

Proposition 5.1 follows from Proposition 5.2 and the following result.

Lemma 5.3. There exists a positive integer $n_{0}$ with the following property. For any finite generating set $S$ of $G$, the inequality $\lambda\left(x, S\left(n_{0}\right)\right)>100 \delta$ holds for all $x \in X$.

Proof. Let $\mathcal{B}$ be a $200 \delta$-separated invariant system of horoballs centered at the parabolic points. Recall that the action of $G$ on $Y(\mathcal{B})$ is proper and cocompact. Let $K \subset Y(\mathcal{B})$ be a compact set such that $\bigcup_{g \in G} g(K)=Y(\mathcal{B})$. Set $a=\operatorname{diam}(K)$ and fix a point $p \in K$. Let $A=\{g \in G: d(g(p), p) \leq 2 a+100 \delta\}$. Notice that $A$ is a finite set. Since $G$ is infinite, there is some $g_{0} \in G$ with $d\left(g_{0}(p), p\right)>100 \delta+2 a$. Notice that if $A$ generates $G$, then

$$
n_{0}:=\max \left\{d_{S}\left(i d, g_{0}\right): S \subset A \text { and } S \text { generates } G\right\}
$$

is finite.

Now let $S$ be a finite generating set of $G$. Suppose there is some $x \in X$ with $\lambda(x, S) \leq 100 \delta$. By the definition of $\lambda(x, S)$, we have $d(s(x), x) \leq 100 \delta$ for all $s \in S$. Recall that $\mathcal{B}$ is $200 \delta$-separated. If $x \in B$ for some $B \in \mathcal{B}$, then $s(B)=B$ for all $s \in S$ and consequently the center of $B$ is fixed by the entire group $G$, a contradiction. Hence $x \in Y(\mathcal{B})$. There is some $\gamma \in G$ with $\gamma(x) \in K$. For $s \in S$, we have $d\left(p, \gamma s \gamma^{-1}(p)\right) \leq d(p, \gamma(x))+d(\gamma(x), \gamma s(x))+d\left(\gamma s(x), \gamma s \gamma^{-1}(p)\right)=d(p, \gamma(x))+$ $d(x, s(x))+d(\gamma(x), p) \leq 2 a+100 \delta$. It follows that $\gamma S \gamma^{-1}:=\left\{\gamma s \gamma^{-1}: s \in S\right\} \subset A$. Clearly $\gamma S \gamma^{-1}$ generates $G$. There is some integer $k, 1 \leq k \leq n_{0}$, and $s_{i} \in S \cup S^{-1}$ $(1 \leq i \leq k)$ such that $g_{0}=\left(\gamma s_{1} \gamma^{-1}\right) \cdots\left(\gamma s_{k} \gamma^{-1}\right)=\gamma\left(s_{1} \cdots s_{k}\right) \gamma^{-1}$. Now

$$
\begin{aligned}
d\left(\gamma^{-1} g_{0} \gamma(x), x\right) & =d\left(g_{0} \gamma(x), \gamma(x)\right) \geq d\left(g_{0}(p), p\right)-d\left(g_{0}(p), g_{0} \gamma(x)\right)-d(\gamma(x), p) \\
& =d\left(g_{0}(p), p\right)-d(p, \gamma(x))-d(\gamma(x), p)>100 \delta+2 a-a-a=100 \delta .
\end{aligned}
$$

Notice $\gamma^{-1} g_{0} \gamma=s_{1} \cdots s_{k} \in S\left(n_{0}\right)$. It follows that $\lambda\left(x, S\left(n_{0}\right)\right)>100 \delta$.

\section{REFERENCES}

[AN] R. Alperin, G. Noskov, Nonvanishing of algebraic entropy for geometrically finite groups of isometries of Hadamard manifolds, preprint(2004).

[B] B. Bowditch, Relatively hyperbolic groups, preprint(1999).

[D] C. Drutu, Quasi-isometric rigidity of groups, preprint(2004).

[EMO] A. Eskin, S. Mozes, H. Oh, Uniform exponential growth for linear groups, Int. Math. Res. Not. 2002, no. 31, 1675-1683. MR.1916428 (2003g:20074)

[F] B. Farb, Relatively hyperbolic groups, Geom. Funct. Anal. 8 (1998), no. 5, 810-840. MR:1650094 (99j:20043)

[G] M. Gromov, Hyperbolic Groups, in "Essays in Group Theory" (ed. S. Gersten) M.S.R.I. Publications No.8, Springer-Verlag(1987) 75-263. MR0919829 (89e:20070)

[GD] R. Grigorchuk, P. de la Harpe, One-relator groups of exponential growth have uniformly exponential growth, translation in Math. Notes 69 (2001), no. 3-4, 575-577. MR.1846003 (2002b:20041)

[GhD] E. Ghys and P. de la Harpe, Sur les groupes hyperboliques d'après Mikhael Gromov, Progress in Mathematics 83.

[HK] G. Hruska, B. Kleiner, Hadamard spaces with isolated flats, Geom. Topol. 9 (2005), 15011538. MR:2175151

[K] M. Koubi, Croissance uniforme dans les groupes hyperboliques, Ann. Inst. Fourier (Grenoble) 48 (1998), no. 5, 1441-1453. MR 1662255 (99m:20080) 
[O1] D. Osin, Relatively hyperbolic groups: Intrinsic geometry, algebraic properties, and algorithmic problems, Mem. Amer. Math. Soc. 179 (2006), no. 843, vi+100pp. MR2182268

[O2] D. Osin, The entropy of solvable groups, Ergodic Theory Dynam. Systems 23 (2003), no. 3, 907-918. MR1992670 (2004f:20065)

[O3] D. Osin, Weakly amenable groups, Contemp. Math., 298 (2002), 105-113.

[W] J. Wilson, On exponential growth and uniformly exponential growth for groups, Invent. Math. 155 (2004), no. 2, 287-303. MR.2031429 (2004k:20085)

[Y] A. Yaman, A topological characterisation of relatively hyperbolic groups, J. Reine Angew. Math. 566 (2004), 41-89. MR.2039323 (2005e:20064)

Department of Mathematical Sciences, University of Cincinnati, Cincinnati, Ohio 45221

E-mail address: xxie@math.uc.edu

Current address: Department of Mathematics, Virginia Tech, Blacksburg, Virginia 24061-0123 